\title{
Axiomatic Characterization of the Median and Antimedian Functions on Cocktail-Party Graphs and Complete Graphs
}

\author{
Manoj Changat* \\ Department of Futures Studies, University of Kerala \\ Trivandrum - 695 034, India \\ e-mail: mchangat@gmail.com \\ Divya Sindhu Lekha \\ Department of Information Technology \\ College of Engineering and Management Punnapra, Alappuzha - 688 003, India \\ e-mail: divi.lekha@gmail.com \\ Henry Martyn Mulder ${ }^{\dagger}$ \\ Econometrisch Instituut, Erasmus Universiteit \\ P.O. Box 1738, 3000 DR Rotterdam, Netherlands. \\ e-mail:hmmulder@few.eur.nl \\ Ajitha R. Subhamathi \\ Department of Computer Applications \\ N.S.S College Rajakumari, Idukki, Kerala, India \\ e-mail:ar.subhamathi@gmail.com
}

24 November 2014

Econometric Institute Report EI 2014-31

This REPORT IS A PREPRINT. IT IS NOT A FORMAL PUBLICATION IN ANY WAY, AND IT WILL BE PUBLISHED ELSEWHERE.

\footnotetext{
${ }^{*}$ Research work is supported by NBHM/DAE under grant No.2/48(2)/2010/NBHM-R \& D

${ }^{\dagger}$ This research was initiated while the third author visited the University of Kerala in January 2011 under the Erudite Scheme of the Government of Kerala, India.
} 


\begin{abstract}
A median (antimedian) of a profile of vertices on a graph $G$ is a vertex that minimizes (maximizes) the remoteness value, that is, the sum of the distances to the elements in the profile. The median (or antimedian) function has as output the set of medians (antimedians) of a profile. It is one of the basic models for the location of a desirable (or obnoxious) facility in a network. The median function is well studied. For instance it has been characterized axiomatically by three simple axioms on median graphs. The median function behaves nicely on many classes of graphs. In contrast the antimedian function does not have a nice behavior on most classes. So a nice axiomatic characterization may not be expected. In this paper an axiomatic characterization is obtained for the median and antimedian functions on complete graphs minus a perfect matching (also known as cocktail-party graphs). In addition a characterization of the antimedian function on complete graphs is presented.
\end{abstract}

Keywords: median, antimedian, consensus function, consistency, cocktail-party graph, complete graph, consensus axiom

AMS subject classification (2000): 05C12, 05C99,05C $38,90 \mathrm{~B} 80$

\title{
1 Introduction
}

Facility location problems in discrete location theory deal with functions that find an appropriate location for a common facility or resource in a discrete network. The main objective is to minimize the cost of accessing a facility or sharing a resource in the network. Placing a common resource at a median position minimizes the cost of sharing the resource with other locations. Thus the algorithms for locating at medians in a graph are very often useful and form the basic models of discrete facility location problems. Typical problems of this kind that have been studied extensively are: (i) The median problem: finding a vertex that minimizes the distance sum to the clients. (ii) The mean problem: finding a vertex that minimizes the sum of the squares of the distances to the clients. (iii) the center problem: minimizing the maximum distance to the clients. The first two problems can be used to model finding the optimal location for a distribution center. The last problem can be used to model finding the optimal location for a fire station. The antimedian problem is a different type of location problem in which the facility is of obnoxious nature (i.e. the clients want to have it as far away as possible), for example a garbage dump. In this case the 'cost' is being maximized.

A consensus function is used to model consensus problems. These are problems in which one wants to reach consensus amongst agents or clients in a rational way. The input of the consensus function is information on the clients and the output concerns the issue on which consensus should be reached. To guarantee the rationality of the process, the consensus function satisfies certain "rational" rules called "consensus axioms". Such axioms should be appealing and simple. But this depends on the consensus function. A function with nice properties might be characterized by simple axioms. But a function that behaves badly might need more complicated or less appealing axioms. K. Arrow 
initiated the study of the axiomatics of consensus functions in his seminal paper [1] of 1951. For more references in this area see [2], [3], [17].

Location problems can also be viewed as consensus problems. Then one wants to characterize the location function by a set of axioms that are as nice and simple as possible. Holzman [10] was the first to study location functions from this perspective. His focus was on the mean function on a tree network (the continuous variant of a tree). Then Vohra [26] characterized the median function axiomatically on tree networks (continuous case). The discrete case was first dealt with by McMorris, Mulder \& Roberts [16]: the median function on cube-free median graphs was characterized using three simple and appealing axioms, see below. The mean function on trees (discrete case) was first characterized by McMorris, Mulder \& Ortega [14], [15]. The center function on trees has been characterized by McMorris, Roberts \& Wang [18], see also [24]. The center function is also studied on some other graph classes, see [27]. Recently the median function has been characterized on hypercubes and median graphs by Mulder \& Novick [22], [23] using the same three simple axioms as in [16]. In the case of the median function and the center function all axioms satisfy the criterion of being appealing and natural at first sight. The characterizations for the mean function are more complex than those for the median function or the center function. But except for one complex axiom they still satisfy the criterion of being simple and appealing. All above results for the center function and the mean function so far are on trees. The characterization for the median function is on a much wider class, viz. that of median graphs. The reason for this is the very nice behavior of the median function on these graphs. For more information on median graphs see e.g. [11], [20], [21].

We focus on the characterization of two location functions: the median function and the antimedian function. The antimedian function maximizes the sum of the distances to the clients, see e.g. [19], [4], [5], [6], [7], [25]. The differences between these two functions are quite striking. A first inspection of the antimedian function already shows that, even on trees, it does not behave nicely at all, let alone on arbitrary graphs. Only on special classes, such as paths, hypercubes and complete graphs, does it seem to have a nice behavior. The axiomatization of the antimedian function on hypercubes and paths is well studied in [8]. In this paper we focus on the cocktail-party graphs. A cocktailparty graph is a complete graph of even order minus a perfect matching. Besides we also study the antimedian function on complete graphs.

In Section 2 we set the stage. In Section 3 we characterize the median function on cocktail-party graphs by a set of four axioms. In Section 4 we characterize the antimedian function on the same graphs by another set of four axioms. In our view they are all simple and natural. In Section 5, we characterize the antimedian function on complete graphs, again by a set of four axioms. For axiomatic characterizations of the median function on complete graphs we refer to [13].

\section{Preliminaries}

Let $G=(V, E)$ be a finite, connected, simple graph with vertex set $V$ and edge set $E$. The distance function of $G$ is denoted by $d$, where $d(u, v)$ is the length of a shortest 
$u, v$-path. The interval $I(u, v)$ between two vertices $u$ and $v$ in $G$ consists of all vertices on shortest $u, v$-paths, that is:

$$
I(u, v)=\{x \mid d(u, x)+d(x, v)=d(u, v)\}
$$

A profile $\pi$ of length $k=|\pi|$ on $G$ is a non-empty sequence $\pi=\left(x_{1}, x_{2}, \ldots, x_{k}\right)$ of vertices of $V$ with repetitions allowed. We define $V^{*}$ to be the set of all profiles of finite length. We call $x_{1}, x_{2}, \ldots, x_{k}$ the elements of the profile. A vertex of $\pi$ is a vertex that occurs as an element in $\pi$. By $\{\pi\}$ we denote the set of all vertices of $\pi$. Note that a vertex may occur more than once as element in $\pi$. If we say that $x$ is an element of $\pi$, then we mean an element in a certain position, say $x=x_{j}$ in the $j$-th position. A subprofile of $\pi$ is just a non-empty subsequence of $\pi$. The concatenation of profiles $\pi$ and $\rho$ is denoted by $\pi \rho$. The profile consisting of the concatenation of $m$ copies of $\pi$ is denoted by $\pi^{m}$. Let $\pi$ be a profile on $G$. A vertex in $\pi$ with highest occurrence in $\pi$ is called a plurality vertex of $\pi$. We denote the set of plurality vertices of $\pi$ by $\operatorname{Pl}(\pi)$.

A consensus function on $G$ is a function $F: V^{*} \rightarrow 2^{V}-\emptyset$ that gives a non-empty subset of $V$ as output for each profile on $G$. For convenience, we write $F\left(x_{1}, \ldots, x_{k}\right)$ instead of $F\left(\left(x_{1}, \ldots, x_{k}\right)\right)$, for any function $F$ defined on profiles, but will keep the brackets where needed.

The remoteness of a vertex $v$ to profile $\pi$ is defined as

$$
r(v, \pi)=\sum_{i=1}^{k} d\left(x_{i}, v\right) .
$$

A vertex minimizing $r(v, \pi)$ is called a median of the profile. The set of all medians of $\pi$ is the median set of $\pi$ and is denoted by $M(\pi)$. A vertex maximizing $r(v, \pi)$ is called an antimedian of the profile. The set of all antimedians of $\pi$ is the antimedian set of $\pi$ and is denoted by $A M(\pi)$. We can also think of $M$ and $A M$ as functions from $V^{*}$ to $2^{V}-\emptyset$, and then call them the Median Function and Antimedian Function. Note that we have

$$
M(x)=\{x\}
$$

and

$$
M(x, y)=I(x, y) .
$$

Moreover, if $I(u, v) \cap I(v, w) \cap I(w, u) \neq \emptyset$, then

$$
M(u, v, w)=I(u, v) \cap I(v, w) \cap I(w, u) .
$$

The median function has been studied extensively, especially on median graphs. A median graph is defined by the property that $|I(u, v) \cap I(v, w) \cap I(w, u)|=1$, for any three vertices $u, v, w$. Equivalently, a median graph is a graph such that any profile of length 3 has a unique median. See e.g. [20], [11], [21] for a rich structure theory on median graphs. Also nice axiomatic characterizations are available for the median function on median graphs, see e.g. [16], [17], [23]. Three simple and natural axioms suffice for the characterization of the median function in this case. We present these here. The first two axioms are defined without any reference to metric. 
(A) Anonymity: $F(\pi)=F\left(x_{\chi(1)}, x_{\chi(2)}, \ldots, x_{\chi(k)}\right)$, for any profile $\pi=\left(x_{1}, x_{2}, \ldots, x_{k}\right)$ on $V$ and for any permutation $\chi$ of $\{1,2, \ldots, k\}$.

(C) Consistency: If $F(\pi) \cap F(\rho) \neq \emptyset$, for profiles $\pi$ and $\rho$, then $F(\pi \rho)=F(\pi) \cap F(\rho)$.

(B) Betweenness: $F(u, v)=I(u, v)$, for all $u, v$ in $V$.

Clearly, the median function satisfies axioms $(A)$ and $(B)$ on any graph. It is part of folklore that the median function also satisfies $(C)$. Anyway, a proof of this can be found in [16].

The cocktail-party graph $K_{(n \times 2)}$ is obtained from the complete graph $K_{2 n}$ with vertex set $V=\left\{v_{1}, \ldots, v_{n}, v_{n+1}, \ldots, v_{2 n}\right\}$ by deleting the perfect matching $v_{1} v_{n+1}, \ldots, v_{n} v_{2 n}$, see e.g. [9]. It arises in the handshake problem. It is distance-transitive, and hence also distance-regular. For each $i$ with $1 \leq i \leq n$, we call $\left\{v_{i}, v_{n+i}\right\}$ a pair of mates. For any vertex $v$, we denote its mate by $\tilde{v}$. For any profile $\pi$, the profile $\tilde{\pi}$ is obtained from $\pi$ by replacing each element by its mate. For $v$ in $V$, the profile $(v, \tilde{v})$ is called a mating pair. A mating profile is the concatenation of mating pairs. Note that, going from left to right through such a profile, each vertex in an odd position is followed by its mate in the next position. Finally, a mate-free profile $\pi$ is such that if $v$ is in $\pi$, then $\tilde{v}$ is not in $\pi$.

The following lemma is obvious but quite helpful in the sequel.

Lemma 1 Let $G$ be a cocktail-party graph with vertex set $V$, and let $\pi=(v, \tilde{v})$ be a mating pair. Then $r(u, \pi)=2$, for all $v$ in $V$.

An immediate consequence of this lemma is that we can compute the median and antimedian function quite simply. Let $\pi$ be a profile on the cocktail-party graph. Assume that $\pi$ contains two elements that form a pair of mates, say $v, \tilde{v}$. Let $\pi^{\prime}$ be the profile obtained from $\pi$ by removing the two elements $v$ and $\tilde{v}$. Consider the remoteness of any vertex $u$ with respect to $\pi$. Now $u$ minimizes (maximizes) $r(u, \pi)$ if and only if it minimizes (maximizes) $r\left(u, \pi^{\prime}\right)$. So we have $M(\pi)=M\left(\pi^{\prime}\right)$ and $A M(\pi)=A M\left(\pi^{\prime}\right)$. Hence, in computing the median set or antimedian set of $\pi$, we can delete any pair of mates. Thus a subprofile $\rho$ remains that is mate-free. Now the median vertices are precisely the vertices with highest occurrence in $\rho$, so $M(\pi)=P l(\rho)$. For this fact we present an argument in the next section. The antimedian vertices are precisely the mates of the vertices with highest occurrence, so $A M(\pi)=P l(\tilde{\rho})$.

\section{Axiomatic Characterization of the Median Func- tion on Cocktail-Party Graphs}

In this section we characterize the median function on cocktail-party graphs. The next two lemmata are presented to put forward two basic properties of the median function. They are the motivation for the two additional axioms besides Anonymity and Consistency that we need for the median function. The first lemma is a trivial consequence of Lemma 1. 
Lemma 2 Let $F$ be the median function defined on the vertex set $V$ of a cocktail-party graph $G$. Then $F(v, \tilde{v})=V$, for any $v \in V$.

The next lemma is also simple.

Lemma 3 Let $F$ be the median function defined on the vertex set $V$ of a cocktail-party graph $G$. Then $F(\pi)=P l(\pi)$, for all mate-free profiles $\pi$.

Proof. Let $\pi=\left(x_{1}, x_{2}, \ldots, x_{k}\right)$ be a mate-free profile. Let $\{\pi\}=\left\{y_{1}, y_{2}, \ldots, y_{\ell}\right\}$, and let $f_{j}$ be the number of occurrences of $y_{j}$ in $\pi$. Then, for any vertex $w$ outside the profile $\pi$, we have $d\left(w, y_{j}\right) \geq 1$, for each vertex $y_{j}$ in $\pi$. Write $f=\sum_{j=1}^{\ell} f_{j}$. So we have $r(w, \pi) \geq f$.

Let $u$ be any vertex in $\pi$. Then we have $d\left(u, x_{i}\right)=1$, for any $x_{i} \neq u$. Clearly $r(u, \pi)=f-f_{j}$, for $u=y_{j}$. So the vertices that minimize remoteness are all in $\pi$. Note that $r(u, \pi)=f-f_{j}$ is minimum when $f_{j}$ is maximum. So the vertices that minimize remoteness are precisely those that occur most often in $\pi$.

By Lemma 2 and Lemma 3, the median function on cocktail-party graphs satisfies the following two axioms.

$\left(A_{1}\right): F(v, \tilde{v})=V$, for all $v \in V$.

$\left(A_{2}\right): F(\pi)=P l(\pi)$, for all mate-free profiles $\pi$.

Let $F$ be any consensus function satisfying the axioms $\left(A_{1}\right)$ and $\left(A_{2}\right)$. Note that for any vertex $v$ in a cocktail-party graph, we have $I(v, \tilde{v})=V$. Now consider any other profile $\pi=(u, v)$ such that $u \neq v, \tilde{v}$. Then, clearly, $u$ and $v$ are adjacent, whence axiom $\left(A_{2}\right)$ implies $F(\pi)=\{u, v\}=I(u, v)$. We put these observations in the following remark.

Remark 4 Let $F$ be a consensus function defined on the vertex set $V$ of a cocktail-party graph $G$ such that $F$ satisfies $A_{1}$ and $A_{2}$. Then $F$ satisfies the Betweenness axiom $(B)$.

Theorem 5 Let $F$ be a consensus function on a cocktail-party graph $G$ with vertex set $V$. Then $F$ is the median function if and only if $F$ satisfies axioms $(A),(C),\left(A_{1}\right)$ and $\left(A_{2}\right)$.

Proof. It is straightforward to check that the median function satisfies all the four axioms.

Let $F$ be a function that satisfies the four axioms. Take any profile $\pi$. If it contains a pair of mates $v, \tilde{v}$, then we can permute $\pi$ such that $v$ and $\tilde{v}$ are moved to the front two positions, thus getting the profile $(v, \tilde{v}) \rho$, where $\rho$ is the subprofile of $\pi$ obtained by deleting the elements $v$ and $\tilde{v}$ from their respective positions. By $\left(A_{1}\right)$, we have $F(v, \tilde{v})=V$. So $F(v, \tilde{v}) \cap F(\rho) \neq \emptyset$. Hence, by Consistency we have $F((v, \tilde{v}) \rho)=$ $F(v, \tilde{v}) \cap F(\rho)=F(\rho)$. Finally, by Anonymity we have $F(\pi)=F(v, \tilde{v}) \cap F(\rho)=F(\rho)$. We can repeat this process until we end up with a subprofile $\sigma$ of $\pi$ that is either a 
mating pair or mate-free. In the latter case, we have $F(\pi)=F(\sigma)$. From axiom $\left(A_{2}\right)$, it follows that $F(\sigma)=P l(\sigma)=M(\sigma)=M(\pi)$. If $\sigma$ is a mating pair, then we have $F(\sigma)=V=F(\pi)=M(\pi)$. This completes the proof.

For any axiomatic characterization, we want to know whether the axioms involved are independent. We present some examples. In all cases $G$ is a cocktail-party graph with vertex set $V$ having at least 4 vertices.

Example 6 (( $\left.A_{1}\right)$ excluded.) Define the function $F$ on $G$ by $F(\pi)=\operatorname{Pl}(\pi)$, for all profiles $\pi$. It is straightforward to check that $F$ satisfies $(A),(C)$ and $\left(A_{2}\right)$. Since $F(v, \tilde{v})=\{v, \tilde{v}\} \neq V$, for any vertex $v$, the function $F$ does not satisfy $\left(A_{1}\right)$.

Example $7\left(\left(A_{2}\right)\right.$ excluded.) Define the function $F$ on $G$ by $F(\pi)=V$, for all profiles $\pi$. Obviously, $F$ satisfies axioms $(A),(C)$ and $\left(A_{1}\right)$. Take any two adjacent vertices $u$ and $v$ in $G$. Then

$$
F(u, v)=V \neq\{u, v\}=P l(u, v) .
$$

So $F$ does not satisfy $\left(A_{2}\right)$.

Example $8((C)$ excluded.) Define the function $F$ on $G$ by

$(c 1): F(v, \tilde{v})=V$, for all vertices $v$ in $V$,

$(c 2): F(\pi)=P l(\pi)$, for all profiles $\pi$ that are not a mating pair.

Clearly, $F$ satisfies $(A),\left(A_{1}\right)$ and $\left(A_{2}\right)$. Take two vertices $u$ and $v$ that are not mates, and let $\pi=(u, \tilde{u}, v, \tilde{v})$. Then, by $(c 2)$, we have

$$
F(\pi)=P l(\pi)=\{u, \tilde{u}, v, \tilde{v}\} \neq V=F(u, \tilde{u}) \cap F(v, \tilde{v}) .
$$

So F does not satisfy Consistency.

The case of Anonymity seems to be different. First we observe that the independence of Anonymity from other axioms is a non-trivial issue for other sets of axioms. In [12] two examples of sets are given where it is highly non-trivial that Anonymity is independent from the other axioms. One instance is the above mentioned case of the set $(A),(B),(C)$ that characterizes the median function on median graphs. A rather intricate example was needed to show independence of $(A)$. In our case we do not yet have an example that shows independence of Anonymity. On the other hand one would not expect that it follows from the other axioms. So we leave it as an open problem here.

\section{Axiomatic Characterization of the Antimedian Func- tion on Cocktail-Party Graphs}

First we present the analogue of axiom $\left(A_{2}\right)$ that we need for the antimedian case. We skip the analogue of Lemma 3 and its proof. An obvious adaptation does the trick.

$\left(A_{3}\right): F(\pi)=P l(\tilde{\pi})$, for all mate-free profiles $\pi$. 
Theorem 9 Let $F$ be a consensus function on cocktail-party graph $G$ with vertex set $V$. Then $F$ is the antimedian function if and only if $F$ satisfies axioms $(A),\left(C\left(,\left(A_{1}\right)\right.\right.$ and $\left(A_{3}\right)$.

Proof. Let $F$ be the antimedian function. Then $F$ satisfies all the above four axioms.

Let $F$ be a function that satisfies the four axioms. Take any profile $\pi$. If it contains a pair of mates $v, \tilde{v}$, then we can permute $\pi$ such that $v$ and $\tilde{v}$ are moved to the front two positions, thus getting the profile $(v, \tilde{v}) \rho$, where $\rho$ is the subprofile of $\pi$ obtained by deleting the elements $v$ and $\tilde{v}$ from their respective positions. By $\left(A_{1}\right)$, we have $F(v, \tilde{v})=V$. So $F(v, \tilde{v}) \cap F(\rho) \neq \emptyset$. Hence, by Consistency, we have $F((v, \tilde{v}) \rho)=$ $F(v, \tilde{v}) \cap F(\rho)=F(\rho)$. Finally, by Anonymity, we have $F(\pi)=F(v, \tilde{v}) \cap F(\rho)=F(\rho)$. We can repeat this process until we end up with a subprofile $\sigma$ of $\pi$ that is either a mating pair or mate-free. In the latter case, we have $F(\pi)=F(\sigma)$. From axiom $\left(A_{3}\right)$ it follows that $F(\sigma)=P l(\tilde{\sigma})=A M(\sigma)=A M(\pi)$. If $\sigma$ is a mating pair, then we have $F(\sigma)=V=A M(\sigma)=A M(\pi)$. This completes the proof.

Again we study the independence of the axioms.

Example $10\left(\left(A_{1}\right)\right.$ excluded.) Define the function $F$ on $G$ by $F(\pi)=P l(\tilde{\pi})$, for all profiles $\pi$. It is straightforward to check that $F$ satisfies $(A),(C)$ and $\left(A_{3}\right)$. Since $F(v, \tilde{v})=\{\tilde{v}, v\} \neq V$, for any vertex $v$, the function $F$ does not satisfy $\left(A_{1}\right)$.

Example $11\left(\left(A_{3}\right)\right.$ excluded.) Define the function $F$ on $G$ by $F(\pi)=V$, for all profiles $\pi$. Obviously, $F$ satisfies axioms $(A),(C)$ and $\left(A_{1}\right)$. Take any two adjacent vertices $u$ and $v$ in $G$. Then

$$
F(u, v)=V \neq\{\tilde{u}, \tilde{v}\}=P l(\tilde{u}, \tilde{v}) .
$$

So $F$ does not satisfy $\left(A_{3}\right)$.

Example $12((C)$ excluded.) Define the function $F$ on $G$ by $(c 1): F(v, \tilde{v})=V$, for all vertices $v$ in $V$, $(c 2): F(\pi)=P l(\tilde{\pi})$, for all profiles $\pi$ that are not a mating pair.

Clearly, $F$ satisfies $(A),\left(A_{1}\right)$ and $\left(A_{3}\right)$. Take two vertices $u$ and $v$ that are not mates, and let $\pi=(u, \tilde{u}, v, \tilde{v})$. Then, by $(c 2)$, we have

$$
F(\pi)=P l(\tilde{\pi})=\{\tilde{u}, u, \tilde{v}, v\} \neq V=F(u, \tilde{u}) \cap F(v, \tilde{v}) .
$$

So $F$ does not satisfy Consistency.

Also in this case we do not have an example yet that shows the independency of Anonymity. Again we leave this as an open problem. 


\section{Axiomatic Characterization of Antimedian Func- tion on Complete Graphs}

In [13] an extensive study is made of location functions on the complete graph that satisfy the above axioms $(A),(B)$ and $(C)$. So we skip this case here.

As can be expected, due to its nice behavior on $K_{n}$, there is also a simple axiomatic characterization of the antimedian function on complete graphs. For the one vertexgraph see above. So let $n>1$, and let $V=\left\{v_{1}, v_{2}, \ldots, v_{n}\right\}$ be the vertex set of $K_{n}$. Note that, in writing $V$ in this way, we have chosen a preferred ordering of the vertices in $V$. Consistent with the approach above we seek axioms besides $(A)$ and $(C)$ that involve as few profiles as possible. Recall that $\{\pi\}$ is the set of vertices occurring in $\pi$. The set $W_{\pi}$ is the set of vertices that occur the least in $\pi$. Note that, if $\{\pi\}$ is a proper subset of $V$, then

$$
W_{\pi}=V-\{\pi\} .
$$

Moreover, if $\pi$ contains all vertices exactly $m$ times, for some $m>0$, then $W_{\pi}=V$. Obviously we have $A M(\pi)=W_{\pi}$. The two axioms we have in mind are

Completeness: $F\left(v_{1}, v_{2}, \ldots, v_{n}\right)=V$.

Complement: $F(x)=V-\{x\}$, for each $x \in V$.

In the Completeness axiom we have only one profile that contains each element of $V$ once and the elements are in the preferred ordering. The Complement axiom involves only profiles containing one element.

Theorem 13 Let $F$ be a consensus function on $K_{n}$ with $n>1$. Then $F$ is the antimedian function if and only if $F$ satisfies $(A),(C)$, Completeness and Complement.

Proof. Clearly the antimedian function satisfies the four axioms.

Conversely, let $F$ satisfy the four axioms. Take a profile $\pi=\left(x_{1}, x_{2}, \ldots, x_{k}\right)$. If $\{\pi\}$ is a proper subset of $V$, then we can write $\pi$ as the concatenation of the singleton profiles $\left(x_{1}\right),\left(x_{2}\right), \ldots,\left(x_{k}\right)$. By Complement the intersection of the sets $F\left(x_{1}\right), F\left(x_{2}\right), \ldots, F\left(x_{k}\right)$ equals $W_{\pi}=V-\{\pi\}$, and by $(C)$ we are done. If all vertices of $V$ occur exactly $m$ times in $\pi$ with $m>0$, then, due to Anonymity, we can write $\pi=\left(v_{1}, v_{2}, \ldots, v_{n}\right)^{m}$, and we are done by $(C)$ and Completeness.

Now let $\pi$ be any other profile. Then there is a number $m>0$ such that some but not all vertices occur exactly $m$ times in $\pi$ whereas the other vertices occur more than $m$ times in $\pi$. Due to Anonymity we can write $\pi=\pi^{\prime}\left(v_{1}, v_{2}, \ldots, v_{n}\right)^{m}$, where $\pi^{\prime}$ is a profile such that $W_{\pi^{\prime}}$ is the set of vertices that occur exactly $m$ times in $\pi$. By the above observations and $(C)$, we have $F(\pi)=F\left(\pi^{\prime}\right) \cap V=W_{\pi^{\prime}}=A M(\pi)$.

Again in this case we do not yet have an example that shows whether Anonymity is independent from the other axioms. The examples below show the independence of the other three axioms. 
Example 14 (Complement excluded.) Let $F$ be defined by $F(\pi)=V$ for all profiles. Then it fails Complement but satisfies trivially the other axioms.

Example $15((C)$ excluded.) Let $F$ be defined by

(k1) $F(x)=V-\{x\}$, for any $x \in V$,

(k2) $F(\pi)=V$, for any profile $\pi$ of length at least 2 .

Then $F$ fails $(C)$ but trivially satisfies the other axioms.

Example 16 (Completeness excluded.) Let $F$ be defined by

(k3) $F(\pi)=\left\{v_{1}\right\}$, for any $\pi$ with $\{\pi\}=V$,

(k4) $F(\pi)=V-\{\pi\}$, for any $\pi$ with $\{\pi\} \neq V$.

Clearly $F$ satisfies $(A)$ and Complement. By $(k 3) F$ fails Completeness. It remains to check Consistency. So let $\pi$ and $\rho$ be two profiles. If $\{\pi\}=V=\{\rho\}$, then

$$
F(\pi)=F(\rho)=F(\pi \rho)=\left\{v_{1}\right\}
$$

and we are done. If $\{\pi\}=V$ and $\{\rho\} \neq V$, then $F(\pi) \cap F(\rho) \neq \emptyset$ only if $\rho$ does not contain $v_{1}$. In this case it again follows that

$$
F(\pi)=F(\pi) \cap F(\rho)=\left\{v_{1}\right\}=F(\pi \rho) .
$$

Finally, let $\{\pi\}$ and $\{\rho\}$ both be proper subsets of $V$. Then $F(\pi)=V-\{\pi\}$ and $F(\rho)=$ $V-\{\rho\}$. These two sets have a non-empty intersection if and only if $\{\pi\} \cup\{\rho\}=\{\pi \rho\}$ is a proper subset of $V$. Again we have $F(\pi \rho)=F(\pi) \cap F(\rho)$.

Open Problem. $(A)$ independent?

In this case we want to elaborate a little more on our trials to find an example. Note that for independence of $(A)$ the ordering of the elements in a profile is essential. We want to split $\pi$ into subprofiles to get a grip on $F(\pi)$. The only subprofiles of $\pi$ that we consider are those containing consecutive elements of $\pi$. So, if we say that $\pi^{\prime}$ is a subprofile of $\pi$, then it is assumed that $\pi^{\prime}$ consists of consecutive elements of $\pi$. As above, a profile is of length at least 1, but for our purposes here, a subprofile now may be empty. We use the convention that $\rho^{m}$ is the empty subprofile if $m=0$. We set $\tau=\left(v_{1}, v_{2}, \ldots, v_{n}\right)$ to be the profile containing each vertex once and in the preferred ordering. Any profile $\pi$ can be written as

$$
\pi=\tau^{m_{0}} \pi_{1} \tau^{m_{1}} \pi_{2} \ldots \pi_{r-1} \tau^{m_{r-1}} \pi_{r} \tau^{m_{r}}
$$

such that

(i) $\pi_{1}, \pi_{2}, \ldots, \pi_{r}$ are non-empty subprofiles of $\pi$ that do not contain $\tau$,

(ii) $m_{1}, m_{2}, \ldots, m_{r-1}>0$ and $m_{0}, m_{r} \geq 0$.

We call this the standard form of $\pi$. If $\pi$ does not contain $\tau$, then we take $r=1$ and $m_{0}=m_{1}=0$. If $\pi=\tau^{m}$ for some $m>0$, then we take $r=0$ and $m_{0}=m$. Note that, if $m_{j}>0$ for some $j$, then $\{\pi\}=V$. Hence, if $\{\pi\} \neq V$, then $\pi$ is the standard form of $\pi$. 
We distinguish two types of profiles.

Type A. $\pi=\tau^{m_{0}} \pi_{1} \tau^{m_{1}} \pi_{2} \ldots \pi_{r-1} \tau^{m_{r-1}} \pi_{r} \tau^{m_{r}}$ with $\cup_{1 \leq j \leq n}\left\{\pi_{j}\right\} \neq V$.

Type B. $\pi=\tau^{m_{0}} \pi_{1} \tau^{m_{1}} \pi_{2} \ldots \pi_{r-1} \tau^{m_{r-1}} \pi_{r} \tau^{m_{r}}$ with $\cup_{1 \leq j \leq n}\left\{\pi_{j}\right\}=V$.

If $\pi$ is of type $A$, then either $\pi=\tau^{m}$ for some $m>0$, in which case $A M(\pi)=V$, or $\pi=\tau^{m_{0}} \pi_{1} \tau^{m_{1}} \pi_{2} \ldots \pi_{r-1} \tau^{m_{r-1}} \pi_{r} \tau^{m_{r}}$ for some $r \geq 1$, in which case

$$
A M(\pi)=V-\left[\cup_{j=1}^{n}\left\{\pi_{j}\right\}\right]=\cap_{j=1}^{n}\left[V-\left\{\pi_{j}\right\}\right] .
$$

Let $F$ be any consensus function satisfying Consistency, Completeness and Complement. Then it follows that we have $F(\pi)=A M(\pi)$, for any profile $\pi$ of type $A$. So the only way to differ from $A M$ is on profiles of type $B$.

Let $\pi$ and $\rho$ be two profiles of type $A$. If the concatenated profile $\pi \rho$ is also of type $A$, then it is straightforward to check that $(C)$ holds. If $\pi \rho$ is of type $B$, then it is straightforward to check that $F(\pi) \cap F(\rho)=\emptyset$, so this does not affect Consistency. Problems may arise when $\pi$ or $\rho$ are of type $B$. Let $\pi$ and $\rho$ be two profiles with $F(\pi) \cap F(\rho) \neq \emptyset$. We write both in standard form:

$$
\begin{aligned}
\pi & =\tau^{m_{0}} \pi_{1} \tau^{m_{1}} \pi_{2} \ldots \pi_{r-1} \tau^{m_{r-1}} \pi_{r} \tau^{m_{r}}, \\
\rho & =\tau^{n_{0}} \rho_{1} \tau^{n_{1}} \rho_{2} \ldots \rho_{s-1} \tau^{n_{s-1}} \rho_{s} \tau^{n_{s}} .
\end{aligned}
$$

Now consider the case that $m_{r}=0$ and $\pi_{r}=\left(v_{1}, v_{2}, \ldots, v_{t}\right)$ and $n_{0}=0$ and $\rho_{1}=$ $\left(v_{t+1}, \ldots, v_{n}\right)$, for some $t$ with $1 \leq t<n$. If we concatenate $\pi$ and $\rho$ and consider the standard form of $\pi \rho$, then $\pi_{r} \rho_{1}=\tau$ and $\pi_{r}$ and $\rho_{1}$ 'disappear'. So they do not count when we want to determine the type of $\pi \rho$. So $\pi \rho$ might be of type $A$, whereas at least one of $\pi$ and $\rho$ is of type $B$. This makes it difficult to assign values to profiles of type $B$. On the other hand, one does not expect that we can deduce Anonymity from the other three axioms.

\section{Concluding Remarks}

The median and antimedian functions satisfy Anonymity and Consistency on any metric space.

On cocktail-party graphs we need two more axioms: in both cases axiom $(A 1)$, for the median case also $(A 2)$, and for the antimedian case also $(A 3)$. All these axioms are natural and intuitively appealing. Except for Anonymity, we have shown independence of the axioms.

Also on the complete graphs we have a simple axiomatic characterization of the antimedian function. We need two more axioms here: Completeness and Complement. Again independence of $(A)$ is an open problem. 


\section{References}

[1] Arrow, K.: Social Choice and Individual Values, In: No. 12 in Cowles Commission for Research in Economics - Monographs, Wiley, New York, First Ed. (1951)

[2] Arrow, K.J.,Sen, A.K., Suzumura K.(eds.): Handbook of Social Choice and Welfare, Volume 1, North Holland, Amsterdam. (2002)

[3] Arrow, K.J.,Sen, A.K., Suzumura K.(eds.): Handbook of Social Choice and Welfare, Volume 1, North Holland, Amsterdam. (2005)

[4] Balakrishnan, K., Brešar, B., Changat, M., Klavžar, S., Imrich, W., Kovše, M., Subhamathi, A.R.: On the Remoteness Function in Median Graphs. Discrete Appl. Math. 157, 3679-3688 (2009)

[5] Balakrishnan, K., Brešar, B., Changat, M., Klavžar, S., Kovše, M., Subhamathi, A.R.: Computing Median and Antimedian Sets in Median Graphs. Algorithmica. 57, 207-216 (2010)

[6] Balakrishnan, K., Brešar, B., Changat, M., Klavžar, S., Kovše, M., Subhamathi, A.R.: Simultaneous Embedding of Graphs as Median and Antimedian Subgraphs. Networks. 56 90-94 (2010)

[7] Balakrishnan, K., Changat, M., Klavžar, S., Joseph, M., Peterin, I., Prasanth, G.N., Špacapan, S.: Antimedian Graphs. Australas. J. Combin., 41 159-170 (2008)

[8] Balakrishnan, K., Changat, M., Mulder, H.M., Subhamathi, A.R.: Axiomatic Characterization of the Antimedian Function on Paths and Hypercubes. Discrete Math. Algorithm. Appl. 04, 1250054, 20 pages (2012)

[9] Deza, M., Laurent, M.: Geometry of Cuts and Metrics. Springer Verlag, Berlin, Heidelberg, New York (1997)

[10] Holzman, R.: An Axiomatic Approach to Location on Networks. Math. Oper. Res. 15 553-563 (1990)

[11] Klavžar, S.,Mulder, H.M.: Median Graphs- Characterizations, Location Theory and Related Structures. J. Combin. Math. Combin. Comput. 30 103-127 (1999)

[12] McMorris, F.R, Mulder, H.M., Novick, B., Powers, R.C.: Five Axioms for Location Functions on Median Graphs. To appear in Discrete Math. Algorithms Appl..

[13] McMorris, F.R, Mulder, H.M., Novick, B., Powers, R.C., Vohra, R.V.: In preparation.

[14] McMorris, F.R, Mulder, H.M., Ortega, O.: Axiomatic Characterization of the Mean Function on Trees. Discrete Math. Algorithms Applications 2 313-329 (2010)

[15] McMorris, F.R, Mulder, H.M., Ortega, O.: Axiomatic Characterization of the $\ell_{p^{-}}$ Function on Trees. Networks. $6094-102$ (2012) 
[16] McMorris, F.R, Mulder, H.M., Roberts,F.S.: The Median Procedure on Median Graphs. Discrete Appl. Math. 84 165-181 (1998)

[17] McMorris, F.R, Mulder, H.M., Vohra, R.V. Axiomatic Characterization of Location Functions. In: Kaul, H., Mulder, H.M. (eds.) Advances in Interdisciplinary Applied Discrete Mathematics, Interdisciplinary Mathematical Sciences Vol. 11, World Scientific Publishing, Singapore, pp. 71-91 (2010)

[18] McMorris, F.R, Roberts,F.S., Wang, C.: The Center Function on Trees. Networks. $38,84-87(2001)$

[19] Minieka, E.: Anticenters and Antimedians of a Network. Networks. 13 35-364 (1983)

[20] Mulder, H.M.: The Interval Function of a Graph. Math. Centre Tracts 132, Math. Centre, Amsterdam, Netherlands (1980)

[21] Mulder, H.M.: Median Graphs. A Structure Theory, In: Kaul, H., Mulder, H.M. (eds.) Advances in Interdisciplinary Applied Discrete Mathematics. Interdisciplinary Mathematical Sciences Vol. 11, World Scientific Publishing, Singapore 93-125 (2010)

[22] Mulder, H.M., Novick, B.A.: An Axiomization of the Median Function on the n-Cube. Discrete Appl. Math. 159 139-144 (2011)

[23] Mulder, H.M., Novick, B.A.: A Tight Axiomatization of the Median Function on Median Graphs. Discrete Appl. Math. 161838 - 846 (2013)

[24] Mulder, H.M., Reid, K.B., Pelsmajer, M.J.: Axiomatization of the Center Function on Trees. Australasian J. Combin. 41 223-226 (2008)

[25] Rao,S.B., Vijayakumar, A.: On the Median and the Antimedian of a Cograph. Int. J. Pure Appl. Math. 46 703-710 (2008)

[26] Vohra, R.: An Axiomatic Characterization of Some Locations in Trees. European J. Operational Research. 90 78-84 (1996)

[27] Shilpa, M., Changat, M., Narasimha-Shenoi, P.G.: Axiomizatic Characterization of the Center Function on Some Graph Classes, Manuscript Submitted. 